\title{
Competition policy, regulation and the institutional design of industry supervision
}

\author{
Cécile Aubert* \\ GREMAQ** \\ Jérôme Pouyet* \\ CORE-UCL and CERAS-ENPC $C^{* * *}$
}

\section{$1 \quad$ Introduction}

The introduction of competition has given rise to important changes in network industries. This is particularly true for telecommunications, in which former protected monopolies have been exposed to competition by new operators in most industrialised countries. Deregulation has also induced entry by new actors, from previously distinct industries (cable, railroads, etc.).

An important dimension of this reorganization process consists in the interaction between regulatory institutions. Indeed, new players are often either unregulated (as for information technologies) or under the jurisdiction of different regulatory bodies (as for broadcasting and telecommunications), although a substantial amount of regulatory oversight is still needed (for interconnection or public service obligations for instance). This raises important issues about the domain of specialization of these multiple regulators, as well as about the coordination of their decisions.

* We are indebted to Jean-Jacques Laffont for stimulating and clarifying discussions during this work. We gratefully acknowledge comments and useful suggestions made by two anonymous referees. The usual disclaimer applies.

** GREMAQ (UMR 5603 CNRS), Manufacture des Tabacs, 21 Allée de Brienne 31000 Toulouse, France.

*** Corresponding author. CORE-UCL, 34 Voie du Roman Pays, 1348 Louvain-la-Neuve, Belgium and CERASENPC (URA 2036 CNRS), 28 rue des Saints-Pères, 75007 Paris, France. Phone number : 32(10)474319. E-mail address : pouyet@core.ucl.ac.be 
Recent research has started to study some of the trade-offs involved in the design of regulatory institutions for these industries. ${ }^{1}$ For telecommunications, the traditional paradigm for regulation is being called into question. Some countries have even proceeded to a (partial) substitution of regulation by competition policy. For instance, New Zealand had decided to give up regulation and to rely solely on competition policy as its new structure for industry supervision, following the Commerce Act of 1986. Experience has shown, however, that this was not sufficient and a Ministerial Inquiry into the regulation of telecommunications, released in September 2000, has put forward the need for more regulation, in order to rule interconnection and to reduce the market power of the incumbent. But economists still cannot provide much guidance on either the transition from regulation to competition policy, or the interaction between these two modes of industry supervision. The specific question that we try to address in this paper is the following: When are the different supervision authorities (i.e., regulation and competition policy in our context) mainly substitutable, or mainly complementary?

Numerous forms of regulation and antitrust enforcement exist. Our attempt therefore hinges on a bare-bone description of the functioning of these institutions. We shall draw upon the following considerations. First, regulatory agencies have wider control rights than antitrust authorities. Indeed, the former engages in detailed regulation of activities whereas the latter assesses ex post the lawfulness of conducts. Second, regulation usually resorts to an ex ante contractual agreement whereas competition policy adopts an ex post monitoring of the industry, even though intervention rules are usually determined ex ante. Third, regulatory agencies, with a narrow mandate, often benefit from a much better knowledge of the industry than antitrust authorities, that have a quasi-universal mandate : regulators engage in a continuing and long-lasting relationship with a specific industry whereas competition policy authorities only intervene in a punctual way.

The framework we adopt can be described as follows. We consider a dominant operator and a fringe producing differentiated (final) goods. The fringe and the dominant operator are taken to have identical efficiency parameters. The fringe is composed of firms that can decide to collude, and therefore act as a monopoly, or to behave competitively, in which case Bertrand-Nash competition takes place in their market.

The dominant operator is supervised by a regulatory agency. This agency offers a contract, which consists in the price of the regulated good and a transfer given to this firm to cover its costs. We study the possibility of complementing regulation with supervision of the fringe, by a competition policy authority. Throughout most of the analysis, we drastically simplify our model by assuming that the competition authority either intervenes - in

1 This literature often considers non-benevolent regulators, following the seminal work of Stigler (1971). This strand of research focuses on the role of institutions on non-benevolent public policy decision-makers (or supervisors in charge of acquiring information). See Laftont and Tirole ((1999), pp. 316-318), for a review of the main insights. 
which case she can deter, at no cost, the fringe from colluding - or does not intervene, and lets the fringe act as a monopolist. Any considerations on the cost of antitrust intervention, or on potential coordination problems (due, say, to conflicting objectives) between regulatory and competition authorities would lessen the benefits of complementing regulation with competition policy. We are thus focusing on the case in which it is the most beneficial, in a partially regulated environment, to add supervision of the fringe to regulation of the dominant operator.

We first build upon the analysis of Laffont and Tirole (1990), (1993), to study how the regulatory agency should depart from standard RamseyBoiteux pricing to account for the fringe's incentive to collude. When the fringe behaves competitively, there is no need to distort the price of the regulated good since the regulator completely internalizes the social cost of the unregulated good and the fringe behaves in a socially optimal way. However, when the fringe colludes, the regulator must distort the provision of the regulated good to implicitly subsidize the collusive fringe in order to give it an incentive to increase its production. With substitutes, this requires to increase the price of the regulated good, whereas with complements, this price has to be decreased in order to increase the demand faced by the fringe.

We show that enforcing a competitive behavior is always welfareenhancing when goods are complements. Indeed, it not only increases consumers' surplus, it also increases the revenue of the regulated firm, thereby reducing the need for distortionary taxation.

This result does not hold with substitutes: when goods are strongly substitutes, enforcing a competitive behavior from the fringe is detrimental to welfare. Indeed, a competitive fringe strongly reduces the revenues from the sale of the regulated good; this requires to increase the subsidy that must be paid to the regulated firm, which has a social cost when public funds are costly. When goods are weak substitutes, antitrust intervention is always beneficial, since it enables to align the fringe's behavior with the socially optimal one, and the loss caused by an increase in distortionary taxation is not too large. Finally, the larger the cost of public funds is, the larger is the degree of substitutability below which it is socially desirable not to enforce competitive pricing by the fringe.

Our model therefore shows that the substitutability/complementarity between supervision authorities strongly depends on the interaction between final product markets.

The outline of the paper is as follows. In the next section (Section 2) we describe our model. We then study in Section 3 the gain obtained by complementing regulation of the dominant operator with competition policy on the fringe. Section 4 presents various extensions of the initial setting. And Section 5 offers some concluding remarks. All proofs are relegated to the Appendix. 


\section{The model}

To avoid confusion, a masculine pronoun will be used for the regulatory agency, and a feminine one for the antitrust authority.

We consider a market for two goods, denoted by ' 1 ' and ' 2 '. Good 1 is produced by a dominant operator, and good 2 by a fringe.

\subsection{Consumers}

Consumers derive a gross surplus from the consumption of quantities $q_{1}$ and $q_{2}$ given by

$$
G S=a\left(q_{1}+q_{2}\right)-\frac{1}{2}\left(q_{1}^{2}+q_{2}^{2}\right)+c q_{1} q_{2}
$$

Parameter $c$ belongs to $(-1,1)$ and indicates whether goods are demand substitutes $(c \leqslant 0)$ or complements $(c \geqslant 0)$.

The telecommunication industry, in which the dominant operator is in charge of fixed telephony, provides us with examples of the two situations : if the fringe is located on the mobile telephony sector, goods are substitutes ${ }^{2}$; if on the other hand the fringe offers internet services, then goods are complementary from the consumers' view point.

The associated demand function for good $i$ is $^{3}$

$$
q_{i}\left(p_{1}, p_{2}\right)=\frac{a(1+c)-p_{i}-c p_{j}}{1-c^{2}}, \quad i \neq j
$$

We omit arguments to ease the presentation, when this cannot lead to confusion. Accordingly, the net surplus derived by consumers is $N S=$ $G S-p_{1} q_{1}-p_{2} q_{2}$.

\subsection{Firms}

A fringe ensures the production of good 2. We assume, first, that the fringe is composed of firms that produce at the same constant marginal cost $\theta$ and, second, that these firms can perfectly coordinate their decisions in order to maximize their joint profits, given by $\pi_{2}=\left[p_{2}-\theta\right] q_{2}$. If the firms choose not to cooperate, then Bertrand competition takes place within the fringe, leading to zero profit in equilibrium. These assumptions are, admittedly,

2 The substitutability between mobile and fixed telephony is a matter of debate among empirical economists, for hardware components. Yet using one or the other type of telephony for each given call remains a choice between substitute products.

3 Throughout the paper, we assume that the size of the market, $a$, is sufficientiy large to ensure positive prices and quantities. Since the second-order conditions associated to the various optimization problems studied in the paper will be always satisfied, this ensures the existence of interior solutions. 
restrictive but will help us focus on our main issue: Is it always welfareenhancing to complement regulation with competition policy?

A dominant operator is in charge of the production of good 1 . We adopt the accounting convention that it receives the revenues from the sales of this good. To abstract from considerations based on a difference of efficiency between the fringe and the dominant operator, we assume that the dominant operator's constant marginal cost is also $\theta$.

This marginal cost of production, $\theta$, is known by both the regulatory agency and the competition policy authority.

Finally, we assume that collusion between the fringe and the dominant operator is not possible. ${ }^{4}$ It is often argued that intra-market collusion is more easily achievable and enforceable than inter-markets collusion, absent any 'external ways' such as mergers or cross-ownership of shares. Besides, in our model, the regulator, who controls the dominant operator, might be able to observe - and thus prevent - any collusive action jointly undertaken by the regulated firm and the unregulated ones.

\subsection{The regulatory agency}

The regulatory agency only controls the dominant operator, and does not regulate the fringe. Examples of such asymmetric regulation abound:In the telecommunications industry for instance, former protected monopolies are usually still under the jurisdiction of regulatory authorities whereas entrants are typically only subject to light regulatory control. We assume in addition that the quantity produced by the fringe is not contractible. The regulator can thus propose a contract $\left\{t_{1}, p_{1}\right\}$ to the regulated firm, where $p_{1}$ is the price required for good 1 and $t_{1}$ is a monetary transfer to compensate this firm for its production cost. The dominant operator's profit function is therefore given by $\pi_{1}=\left[p_{1}-\theta\right] q_{1}+t_{1}-F$, where $F$ is a fixed cost of production ${ }^{5}$.

We denote by $\lambda>0$ the shadow cost of public funds. In our partial equilibrium approach, $\lambda$ captures the fact that taxation is distortionary: transferring one unit of money to the regulated firm requires to levy $1+\lambda$ units from taxpayers ${ }^{i}$.

The welfare function is thus given by

$$
\begin{aligned}
S W & =N S-(1+\lambda) t_{1}+\pi_{1}+\pi_{2} \\
& =G S+\lambda p_{1} q_{1}-(1+\lambda)\left(\theta q_{1}+F\right)-\theta q_{2}-\lambda \pi_{1}
\end{aligned}
$$

4 See Aubert and Pouyet (1999) for a model of incomplete regulation of a dominant firm and potential collusion between this tirm and an unregulated competitor, under asymmetric information.

5 This fixed cost of production justifies the monopoly situation of the dominant firm for good 1 , but it has no qualitative impact on our results, as will be clear in the following. In particular, the justifications we obtain for favoring - or preventing - antitrust intervention are independent from it.

6 We would have obtained similar results if we had assumed that the regulator collects the revenues associated to the sales of good 1, and then pays the regulated firm a transfer. Similarly, if there was no fixed cost, the subsidy would become a tax, and our results could be immediately reinterpreted. 


\subsection{The timing}

The competition policy authority intervenes first; the regulatory agency then contracts with the dominant operator and determines the price for good 1 ; finally, the fringe chooses the price of good 2.7

\subsection{The competition policy authority}

Given its incompleteness, the regulation of the dominant operator might be complemented with supervision of the fringe. We assume that an antitrust authority has access to a costless monitoring technology that enables her to investigate the fringe's behavior and to determine whether anti-competitive actions have taken place.

Our goal is not to determine the optimal antitrust intervention policy but, rather, to study how competition policy impacts the regulatory process. Therefore, we drastically simplify the competition authority's problem by considering that, if she intervenes, then she can discover collusion by the fringe at no cost and punish heavily the firms. Thus, the first stage in our model simply consists in determining whether enforcing a non-cooperative behavior from the fringe is better than letting collusion occur ${ }^{8}$. We shall therefore consider the following two situations :

- Under 'antitrust intervention', the fringe is deterred from colluding and behaves as a price-taker:

$$
p_{2}^{n c}=\theta
$$

where superscript ' $n c$ ' stands for 'non collusive fringe'.

- Under 'no antitrust intervention', the fringe has an incentive to collude in order to maximize joint profits :

$$
p_{2}^{c}=\frac{a+\theta+c\left(a-p_{1}\right)}{2},
$$

where superscript ' $c$ ' stands for 'collusive fringe'.

\section{$3 \quad$ Optimal industry supervision}

Let $p_{2}\left(p_{1}, \theta\right)$ be the best-response of the (Stackelberg follower) fringe. Since there is complete information, the regulatory contract $\left\{t_{1}, p_{1}\right\}$ offered to

7 We comment on this assumption in Section 4.

8 Suppose that the antitrust authority possesses a monitoring technology that enables to detect collusion when she intervenes on the fringe; suppose that her marginal cost of intervention is null for a given industry. Then, she can intervene with probability 1 and totally deter collusion if the fine imposed in case of collusion is sufficiently large; or she can let collusion occur when she does not intervene. 
the dominant operator only has to ascertain that this firm is willing to participate, or ${ }^{9}$

$$
I R_{1}(\theta): \pi_{1}=\left[p_{1}-\theta\right] q_{1}\left(p_{1}, p_{2}\left(p_{1}, \theta\right)\right)+t_{1}-F \geqslant 0
$$

Since the dominant operator's profit is socially costly (see Equation (2)), the regulatory agency will set the transfer $t_{1}$ so that the regulated firm earns no rent : $I R_{1}(\theta)$ is binding in equilibrium. Optimizing social welfare with respect to price $p_{1}$ yields the following first-order condition :

$$
\frac{\partial S W}{\partial q_{1}}\left[\frac{\partial q_{1}}{\partial p_{1}}+\frac{\partial q_{1}}{\partial p_{2}} \frac{\partial p_{2}}{\partial p_{1}}\right]+\frac{\partial S W}{\partial q_{2}}\left[\frac{\partial q_{2}}{\partial p_{1}}+\frac{\partial q_{2}}{\partial p_{2}} \frac{\partial p_{2}}{\partial p_{1}}\right]+\frac{\partial S W}{\partial p_{1}}=0
$$

As can be seen from Equation (5), different effects guide the choice of the price for good 1 by the regulatory agency. Their magnitude depends on the degree of competition within the fringe.

\subsection{Antitrust intervention : competitive fringe}

If the competition policy authority decides to intervene, the fringe is deterred from colluding and $p_{2}=\theta$. This obviously implies that $\frac{\partial p_{2}}{\partial p_{1}}=0$. Moreover, since the fringe behaves in a socially efficient way, there is no need for the regulator to alter the provision of good 1 to affect that of good 2 (i.e., $\frac{\partial S W}{\partial q_{2}}=p_{2}-\theta=0$ ). Thus, in the case of a competitive fringe, Equation (5) reduces to the standard Ramsey-Boiteux formula

$$
\frac{p_{1}^{n c}-\theta}{p_{1}^{n c}}=\frac{\lambda}{1+\lambda} \frac{1}{\eta_{1}}
$$

where $\eta_{1}$ is the price elasticity of good 1 , i.e., $\eta_{1}=-\frac{\partial q_{1}}{\partial p 1} \frac{p_{1}}{q_{1}}$. When the fringe behaves competitively, the choice of the regulated price is only affected by the direct effect of $p_{1}$ on welfare. Since transfers are socially costly, the regulatory agency must distort the price of good 1 away from its marginal cost in order to reduce the subsidy given for cost-reimbursement purposes.

Equations (3) and (6) yield the equilibrium prices $\left\{p_{1}^{n c}, p_{2}^{n c}\right\}$ and enable to obtain the corresponding quantities $\left\{q_{1}^{n c}, q_{2}^{n c}\right\}$, which are explicitly given in the Appendix.

\subsection{No antitrust intervention : collusive fringe}

In this case, the collusive fringe acts as a monopolist on its own market. The regulatory agency would like to subsidize the fringe so as to induce

9 The outside opportunity of a regulated firm that would reject the regulatory contract is normalized to 0 . 
a larger production of good 2. The only tool he can use is a reduction of the demand for good 1 when goods are substitutes, or, equivalently, in this case, an increase in $p_{1}$. Similarly, for complementary goods, the regulatory agency can only use a decrease in $p_{1}$, so as to increase the demand for good 1 , and therefore for good 2.

However, since the regulatory agency acts as the Stackleberg leader of our game, the effect of $p_{1}$ on the price chosen by the fringe also has to be taken into account: when goods are demand substitutes, there exists a countervailing incentive since the increase in $p_{1}$ triggers an increase $\mathrm{e}^{10}$ in $p_{2}$ that finally leads to a decrease in $q_{2}$. Similarly, with demand complements, decreasing $p_{1}$ leads to an increase in $p_{2}$ and therefore to a decrease in the demand for good 2 . In our model, the first effect dominates since

$$
\frac{\partial q_{2}}{\partial p_{1}}+\frac{\partial q_{2}}{\partial p_{2}} \frac{\partial p_{2}}{\partial p_{1}}=\frac{-c}{2\left(1-c^{2}\right)}
$$

The regulatory agency thus increases (decreases) $p_{1}$ when goods are substitutes (complements), so as to account for the incentive of the collusive fringe to reduce its production.

Similarly, the choice of the price of the regulated good 1 must now account for the indirect effect of $p_{2}$ on $q_{1}$. When the fringe colludes, the price of good 1 tends to be larger since this always leads to an increase in $q_{1}$ through the reaction of the fringe (i.e., $\frac{\partial q_{1}}{\partial p_{2}} \frac{\partial p_{2}}{\partial p_{1}}=\frac{c^{2}}{2\left(1-c^{2}\right)} \geqslant 0$ ).

One obtains the following rewriting of the first-order condition associated to $p_{1}$ when the fringe colludes:

$$
\frac{p_{1}^{c}-\theta}{p_{1}^{c}}=\frac{p_{1}^{n c}-\theta}{p_{1}^{n c}}+\frac{1}{(1+\lambda)\left(2-c^{2}\right)}\left[c^{2} \frac{\lambda}{\eta_{1}}-2 \frac{p_{2}^{c}-\theta}{p_{1}^{c}}\right]
$$

The distortion in the price relative to marginal cost is similar to the standard Ramsey-Boiteux formula, but two additional effects arise, as can be seen from the additional components in Equation (7) : first, the regulator takes into account the fact that the price of good 1 influences the collusive price for good 2, from (4), and therefore welfare; second, he tends to compensate for the margin added to marginal $\operatorname{cost} \theta$ by the collusive fringe when it sets the price of good 2. The two effects are weighted according to the degree of complementarity and to the cost of public funds.

Equilibrium prices $\left\{p_{1}^{c}, p_{2}^{c}\right\}$ and quantities $\left\{q_{1}^{c}, q_{2}^{c}\right\}$ are given in the Appendix.

10 Prices are strategic complements with demand substitutes. 


\subsection{Comparison}

Let us now consider the decision by the antitrust authority, in the first period, to intervene or to let collusion occur.

Proposition 1 With substitutes,

- the price of the regulated good is larger when the fringe colludes than when it behaves competitively,

- the regulated dominant operator produces more when the fringe colludes than when it behaves competitively.

With complements, the reverse results hold.

Proof See Appendix A.

口

First, the behavior of the fringe directly affects the demand for the regulated good : since a collusive fringe sets a higher price than a competitive one, collusion increases the demand for the regulated good 1 when goods are substitutes --and decreases it with complements.

Second, as explained previously, the regulator takes into account the incentive of the collusive fringe to under-produce: he tends to increase the price of good 1 with substitutes, and to decrease it with complementary outputs. This gives rise to an effect, on the demand for the regulated good, that goes against the previous one.

To pave the road for the next proposition, let us now consider the revenue earned from the regulated good. More specifically, let us assume for the moment that the shadow cost of public funds is very large. Then, we obtain

$$
\lim _{\lambda \rightarrow+\infty}\left\{\left[p_{1}^{n c}-\theta\right] q_{1}^{n c}-\left[p_{1}^{c}-\theta\right] q_{1}^{c}\right\}=\frac{c(1+c)(4-3 c)}{8(1-c)\left(2-c^{2}\right)}(a-\theta)^{2},
$$

which is negative with substitutes, and positive with complements. This means that when goods are substitutes, the net revenue generated from the sale of the regulated good is larger when the fringe colludes than when it competes. As a consequence, still with substitutable goods, the subsidy that must be paid to the dominant operator is larger when the fringe behaves competitively than when it colludes. In our model, this is not welfareneutral since taxation is distortionary. And the deadweight loss associated to this subsidy increases with the cost of public funds. A reverse conclusion arises with complements : enforcing a competitive behavior from the fringe increases the revenue associated to the sale of the regulated production, which in turns alleviates the need for distortionary taxation.

The case of an infinite shadow cost of public funds is obviously unrealistic. The following proposition applies for finite values of $\lambda$.

Proposition 2 There exists a unique threshold $c^{\prime}(\lambda) \in(-1,0)$ such that

- the competition policy authority does not intervene and lets the fringe collude if $c \leqslant c^{\prime}(\lambda)$, 
- the competition policy authority intervenes and prevents the fringe from colluding if $c \geqslant c^{\prime}(\lambda)$.

Moreover, the threshold $c^{\prime}(\lambda)$ is increasing in $\lambda \geqslant 0$.

Proof See Appendix B.

The two effects at work are the following. First, enforcing a noncooperative behavior from the fringe always increases consumer surplus since the fringe then behaves in a socially optimal way. Second, competition by the fringe affects the transfer that must be paid to the regulated firm. Indeed, when goods are substitutes, the competitive behavior of the fringe leads to an increase in this transfer and, thus, to a distortion due to the social cost of taxation. When goods are complements, on the other hand, a competitive behavior decreases the need for such distortionary taxation : a competitive fringe produces a larger quantity, which raises consumers' willingness to pay for the regulated good, and therefore reduces the transfer given to the regulated dominant operator.

The two effects go in the same direction and contribute to making antitrust intervention desirable for complements. But they go against one another when goods are substitutes.

These results can be contrasted to the ones for standard Cournot competition, without regulation:In such a framework, one would rather tolerate collusion when goods are complements. Indeed, with complements, collusion enables firms to internalize the positive externalities arising from reductions in prices, leading to larger price reductions compared to the competitive price under Cournot competition. To the contrary, antitrust intervention would be more needed when goods are substitutes, since collusion then leads to higher prices. A major difference between our framework and the 'standard' one is that collusion does not take place between firms producing differentiated goods - the regulated monopoly does not collude with the fringe. Since the regulator maximizes consumer surplus, the effects of price reductions are internalized even when there is no collusion between the fringe and the dominant firm.

\section{Discussion}

We examine below whether our results are robust to various assumptions.

- Cournot competition: Let us consider that there is Cournot competition between markets. Assume first that if the antitrust authority intervenes then the fringe still sets a price equal to its marginal cost. Our insights carry over to that situation ${ }^{11}$ : if products are sufficiently substitutable, then it becomes socially preferable to let the fringe collude; otherwise,

11 Proofs are available from the authors upon request. 
antitrust intervention on the fringe is socially desirable. Although the value of the threshold is different, the nature of the competition between markets has little impact on our results. The intuitions remain the same. Assume now that if the fringe behaves competitively, then Cournot competition also takes place within the fringe; in that case the price set by a competitive fringe would be different from the fringe's marginal cost and would no longer be socially efficient. The larger the number of firms in the fringe becomes, the larger is the increase in consumers' surplus generated by competition; However, an increase in the number of firms also reinforces the impact of competition on the size of the subsidy paid to the dominant operator. The complexity of the mathematical expressions make it difficult to obtain a formal result; simple simulations indicate that, in our framework, when the number of firms in the fringe increases, the zone of parameter values such that antitrust intervention is socially preferable, shrinks.

- Intermediate forms of antitrust intervention: The way we have formalized the intervention of the competition policy authority is rather crude. One might for instance consider that the authority can commit to a certain probability of intervention. To keep the modeling as simple as possible, let us consider that the fringe's reaction function is given by : ${ }^{12}$

$$
p_{2}=\frac{a(1+c) \alpha+\theta-c \alpha p_{1}}{1+\alpha}
$$

We acknowledge that this is a rather ad hoc way of modeling antitrust intervention. Parameter $\alpha$ is nevertheless a short-cut to model the intensity of antitrust intervention. If $\alpha=0$ then the fringe behaves competitively; if $\alpha=1$ the fringe acts as a monopolist; the price set by the fringe is increasing with $\alpha$. We denote by $\tilde{\alpha}$ the value of the antitrust intervention parameter that maximizes social welfare, which is decided prior to the regulatory contract. Then, we obtain the following corollary.

Corollary 1 Consider that the fringe's reaction function is given by (8). Then, the optimal antitrust intervention policy is defined as follows :

- When products are strong substitutes, the competition authority lets the fringe collude (i.e., $\tilde{\alpha}=1$ ).

- When products are weak substitutes, the competition authority allows some collusion (i.e., $\tilde{\alpha} \in(0,1)$ ).

When products are complements, the competition authority completely deters the fringe from colluding (i.e., $\tilde{\alpha}=0$ ).

Proof See Appendix C.

Let us focus on the case of weak substitutes. $\tilde{\alpha}$ is decreasing with $c:$ the weaker the substitutability is, the weaker is the impact of the

12 This is obtained when the first-order condition characterizing the collusive fringe's reaction function is $\left(p_{2}-\theta\right) \frac{\partial q_{2}}{\partial p_{2}}+\alpha q_{2}=0$. 
fringe's behavior on the dominant operator's revenue, and the less socially profitable it is to let the fringe collude. For weak substitutes, the antitrust authority accepts an intermediate level of collusive behavior at the level of the fringe.

- Simultaneous timing: In that case, the regulator would no longer be able to alter the fringe's production decision. Notice that this is already the case when the fringe behaves competitively since it prices at marginal cost, whatever the regulated firm's price. Therefore, the only change concerns the case of a collusive fringe. Not surprisingly, our results still carry over to this case. The interesting difference is that the threshold (above which the fringe is deterred from colluding) is smaller under simultaneous timing than under sequential timing. The intuition is that the regulator cannot influence the decisions taken by the fringe; antitrust intervention is therefore more desirable since it can counter the incentive of the fringe to under-produce under collusion.

- Asymmetric information: Incomplete information from the side of the regulator also leaves our results qualitatively unchanged. ${ }^{13}$ The rent extraction phenomenon that arises under incomplete information is somewhat orthogonal to our focus. Qualitative results are unchanged; indeed, competition on the fringe reduces the rent of the firm when goods are substitutes, and increases it when goods are complements.

\section{Conclusion}

We have tried to highlight in our model some of the effects that may guide the design of an optimal industry supervision. The most striking result is that antitrust intervention might not always enhance welfare in partially regulated environments, even when there are no coordination problem between regulatory and competition policy authorities, and when antitrust intervention is costless. Moreover, our model highlights a new insight : the nature of the interaction between final goods affects the nature of the interaction between supervision authorities.

With complementary products, antitrust intervention always increases welfare, through two channels : the increase in consumers' surplus, and the reduction in distortionary taxation. Thus, in presence of complementarities, a rationale arises for a complete supervision of all the firms active in the industry (fringe and dominant operator in our model).

A question that then arises is the following : In our model, could antitrust intervention replace regulatory oversight of the dominant operator? The answer will be negative in general ${ }^{14}$. Indeed, antitrust enforcement

13 Computations are available at www.enpc.fr/ceras/pouyet.

14 For instance, assume that $F=0$ and $\lambda=0$. Then one could check immediately that regulation of the dominant operator only is always preferred to antitust intervention on the fringe only. The (obvious) reasons 
cannot counter the tendency to under-produce of the dominant operator; at most, it can deter the fringe from colluding (and possibly deter the dominant operator from colluding with the fringe), but this precisely reinforces the market power of the dominant operator when outputs are complementary. Finally, in a similar framework to the one used in this paper, Pouyet and Verouden (2001) study the optimal antitrust intervention policy when none of the markets are monopolized. They find that, when goods are strongly complementary, the competition policy authority is forced to intervene with probability 1 in both markets, highlighting the inability of competition authorities (in the traditional vision) to cope alone with markets exhibiting a pattern of strong complementarities.

On the other hand, in presence of strong substitutes, enforcing a competitive behavior would put a strong competitive pressure on an unregulated dominant operator. One would then like to compare the following situations : regulation of the dominant operator only, or antitrust intervention on the fringe only. Such a comparison relies on exogenous parameters (the cost of public funds on one hand, and the cost of antitrust intervention on the other hand) and clearly calls for a deeper understanding of the functioning of these two institutions.

This paper constitutes a first step towards such a better understanding. But much remains to be done in order to answer the daunting questions that arise when one considers the interactions between antitrust and regulation. In particular, why are there two different bodies instead of a regulator also endowed with auditing capacities ? Separation cannot be justified ${ }^{15}$ in our simple framework, except for reasons based on bounded rationality. Assuming that the authorities can have private objectives allows to obtain more complex justifications; separation may indeed be optimal when there is a risk of capture of the authorities by the industry (see Laffont and Martimort (1999)). Another interesting question is the following: why are antitrust authorities generally given a different mandate than regulatory ones? Again, in our framework, there would be no justification for that. Spulber and Besanko (1992) offer a rationale for asking antitrust authorities to take only into account consumer surplus, and not firms profits. In their framework, giving this objective to the antitrust authority is a way of committing to an (ex post inefficient) intervention in order to deter collusion. But the introduction of a regulated transfer financed through distortionary taxation increases the cost of distorting the objective of the competition authority in our model, especially when the cost of public funds is large (for example in

are the following. First, the regulator possesses a broader set of instruments than the antitrust authority. Second, he can intervene ex ante (that is, before the production decisions are undertaken at the industry's level), and therefore use this Stackeiberg leadership to account for the interaction between the dominant operator's and the fringe's decisions. When the shadow cost of public funds is not too large (a realistic assumption for many developed countries), this reasoning still holds. 
developing countries) ${ }^{16}$. These main questions constitute promising avenues for further research.

\section{Appendices}

\section{A - Proof of Proposition 1}

We have

$$
\begin{array}{ll}
p_{1}^{n c}=\frac{\theta+\lambda[a(1+c)+\theta(1-c)]}{1+2 \lambda} & q_{1}^{n c}=\frac{1+\lambda}{(1+2 \lambda)(1-c)}(a-\theta), \\
p_{2}^{n c}=\theta & q_{2}^{n c}=\frac{1+\lambda(2-c)}{(1+2 \lambda)(1-c)}(a-\theta) .
\end{array}
$$

Similarly, we have

$$
\begin{aligned}
& p_{1}^{c}=\frac{a(1+c)[2(2-c) \lambda-c]+[4(1+\lambda)+c(1-2 \lambda-2 c(1+\lambda)]] \theta}{4-3 c^{2}+4\left(2-c^{2}\right) \lambda} \\
& p_{2}^{c}=\frac{1}{2}\left[a(1+c)+\theta-c \frac{a(1+c)(2(2-c) \lambda-c)+[4(1+\lambda)+c(1-2 \lambda-2 c(1+\lambda))] \theta}{4-3 c^{2}+4\left(2-c^{2}\right) \lambda}\right]
\end{aligned}
$$

and

$$
\begin{aligned}
& q_{1}^{c}=\frac{\left(4+c^{3}\right)(1+\lambda)-c(1+2 \lambda)-c^{2}(3+2 \lambda)}{(1-c)\left(4-3 c^{2}+4\left(2-c^{2}\right) \lambda\right)}(a-\theta) \\
& q_{2}^{c}=\frac{2(1+2 \lambda)-2 c \lambda-c^{2}(1+\lambda)}{(1-c)\left(4-3 c^{2}+4\left(2-c^{2}\right) \lambda\right)}(a-\theta)
\end{aligned}
$$

Straightforward computations show that

$$
p_{1}^{n c}-p_{1}^{c}=\frac{c(1+c)}{(1+2 \lambda)} \frac{1+\lambda(4-3 c+4(1-c) \lambda)}{4-3 c^{2}+4\left(2-c^{2}\right) \lambda}(a-\theta)
$$

The sign of this expression only depends on the sign of $c$.

Straightforward computations also show that

$$
q_{1}^{n c}-q_{1}^{c}=c \frac{a-\theta}{(1-c)(1+2 \lambda)\left(4-3 c^{2}+4\left(2-c^{2}\right) \lambda\right)} P_{1}(\lambda),
$$

where $P_{1}(\lambda) \equiv(1+2 \lambda)^{2}+c \lambda-c^{2}(1+\lambda)(1+2 \lambda)$. We have $P_{1}^{\prime}(\lambda)=$ $4(1+2 \lambda)+c-c^{2}(3+4 \lambda) \geqslant 0 \forall \lambda \geqslant 0$ and $P_{1}(0)=1-c^{2}>0$; thus,

Our simple model could be extended to take into account different objectives for the two authorities, but there would be no justification for these objectives. In addition, this extension would require ad hoc assumptions on whether consumers are also taxpayers - in which case the major effects we have underlined in this paper should remain : there would still be a trade-off between increasing consumer surplus and limiting distortionary taxation, for substitute goods. 
$P(\lambda)>0, \forall \lambda>0$. The sign of $q_{1}^{n c}-q_{1}^{c}$ therefore only depends on the sign of $c$.

\section{B - Proof of Proposition 2}

Let $S W^{n c}$ and $S W^{c}$ be the welfare levels when the fringe behaves competitively and when it colludes respectively. Their difference is

$$
S W^{c}-S W^{n c}=\frac{(1+c)(a-\theta)^{2}}{2(1-c)(1+2 \lambda)\left(4-3 c^{2}+4\left(2-c^{2}\right) \lambda\right)} P_{2}(\lambda)
$$

where $P_{2}(c) \equiv-(1+2 c \lambda)(1+2 \lambda)^{2}+c^{2}\left(1+4 \lambda+8 \lambda^{2}+6 \lambda^{3}\right) . P_{2}($.$) admits$ two roots given by

$$
\left\{\begin{array}{l}
\frac{1}{-\lambda-\frac{(1+\lambda) \sqrt{1+2 \lambda+4 \lambda^{2}}}{1+2 \lambda}} \in[-1,0), \forall \lambda \geqslant 0, \\
\frac{1}{-\lambda+\frac{(1+\lambda) \sqrt{1+2 \lambda+4 \lambda^{2}}}{1+2 \lambda}} \geqslant 1, \forall \lambda \geqslant 0
\end{array}\right.
$$

Denote by $c^{\prime}(\lambda)$ the negative root : for all $c \in\left(-1, c^{\prime}(\lambda)\right], S W^{c}-S W^{n c} \geqslant 0$ and for all $c \in\left[c^{\prime}(\lambda), 1\right), S W^{c}-S W^{n c} \leqslant 0$. Finally, it is trivial to show that $c^{\prime}($.$) is an increasing function of \lambda \geqslant 0$ and that $c^{\prime}(0)=-1$.

\section{C - Proof of Corollary 1}

Given the fringe's reaction function, we have :

$$
\frac{\partial S W}{\partial \alpha} \propto \frac{c \lambda(1+\lambda)+\alpha(1-c)\left[1+2 \lambda+c(1+\lambda)^{2}\right]}{\left[-1-2 \lambda+\alpha\left(-1-2 \lambda+c^{2}(1+\lambda)\right)\right]^{3}}
$$

The denominator is always negative.

When products are complements, we have $\frac{\partial S W}{\partial \alpha}<0$.

Consider now that products are substitutes.

$\tilde{\alpha}=\frac{-c \lambda(1+\lambda)}{(1-c)\left[1+2 \lambda+c(1+\lambda)^{2}\right]}$ can be an interior solution provided that $\left.\frac{\partial^{2} S W}{\partial \alpha^{2}}\right|_{\alpha=\tilde{\alpha}} \leqslant 0$ and $\tilde{\alpha} \in[0,1]$. A necessary and sufficient condition for $\tilde{\alpha} \geqslant 0$ is $c \geqslant-\frac{1+2 \lambda}{c(1+\lambda)^{2}}$. A necessary and sufficient condition for $\tilde{\alpha} \leqslant 1$ is $c \geqslant \tilde{c}(\lambda)$, with $0 \geqslant \bar{c}(\lambda) \geqslant-\frac{1+2 \lambda}{c(1+\lambda)^{2}}$.

Consider first that the second-order condition is satisfied or $1+2 \lambda-$ $c^{2}(1+\lambda)^{2} \geqslant 0$. Consider now that $c \in\left[-\frac{1+2 \lambda}{c(1+\lambda)^{2}}, \tilde{c}(\lambda)\right]$. Then, the secondorder condition is satisfied but we must have $\tilde{\alpha}=1$.

Now consider that $(1+2 \lambda)-c^{2}(1+\lambda)^{2}<0$; this implies that $1+2 \lambda+c(1+\lambda)^{2}<0$ and that $\frac{\partial S W}{\partial \alpha}>0$. 


\section{References}

Aubert, C. and J. Pouyet (1999), Incomplete Regulation and Collusion, mimeo.

Brainard, S.L. and D. Martimort (1996), "Strategic Trade Policy Design with Asymmetric Information and Public Contracts", Review of Economic Studies, 63, pp. 81-105.

Brainard, S.L. and D. Martimort (1997), "Strategic Trade Policy with Incompletely Informed Policymakers, Journal of International Economics, 42, pp. 33-65.

Combes, P.P., Caillaud and B. Jullien (1997), "Common Market with Regulated Firms", Annales d'Economie et de Statistiques, 47, pp. 6599.

Green, J. and J.J. Laffont (1977), "Characterization of Satisfactory Mechanisms for the Revelation of Preferences for Public Goods", Econometrica, 45, pp. 427-35.

Laffont, J.J. and D. Martimort (1999), "Separation of Regulators Against Collusive Behavior", Rand Journal of Economics, 30(2), pp. 232-262.

Laffont, J.J. and J. Tirole (1990), "The Regulation of Multiproduct firms. II : Applications and Policy Analysis", Journal of Public Economics, 43 , pp. 37-66.

Laffont, J.J. and J. Tirole (1993), A Theory of Incentives in Procurement and Regulation, Boston, MIT Press.

Laffont, J.J. and J. Tirole (2000), Competition in Telecommunications, MIT Press.

Myerson, R. (1979), "Incentive Compatibility and the Bargaining Problem", Econometrica, vol. 47, pp. 61-73.

Pouyet, J. and V. Verouden (2001), Antitrust Enforcement Policy and Markets Interaction: Targeted or Concerted Interventions ?, mimeo.

Spulber, D. and D. Besanko (1992), "Delegation, Commitment and the Regulatory Mandate", Journal of Law, Economics, and Organization, 8, pp. 126-154.

Stigler, G. (1971), "The Economic Theory of Regulation", Bell Journal of Economics, 2, pp. 3-21. 